\title{
Selective interference of grasp and space representations with number magnitude and serial order processing
}

\author{
Jean-Philippe van Dijck • Wim Fias • Michael Andres
}

Published online: 13 December 2014

(C) Psychonomic Society, Inc. 2014

\begin{abstract}
It has been proposed that the metrics of space, time and other magnitudes relevant for action are coupled through a generalized magnitude system that also contribute to number representation. Several studies capitalized on stimulusresponse compatibility effects to show that numbers map onto left-right representations and grasp representations as a function of their magnitude. However, the tasks typically used do not allow disentangling magnitude from serial order processing. Here, we devised a working memory (WM) task where participants had to remember random sequences of numbers and perform a precision/whole-hand grip (Experiment 1) or a uni-manual left/right button press (Experiment 2) in response to numbers presented during the retention interval. This task does allow differentiating the interference of number magnitude and serial order with each set of responses. Experiment 1 showed that precision grips were initiated faster than wholehand grips in response to small numbers, irrespective of their serial position in WM. In contrast, Experiment 2 revealed an advantage of right over left button presses as serial position increased, without any influence of number magnitude. These findings demonstrate that grasping and left-right movements overlap with distinct dimensions of number processing. These findings are discussed in the light of different theories explaining the interactions between numbers, space and action.
\end{abstract}

J.-P. van Dijck $(\bowtie) \cdot$ W. Fias $\cdot$ M. Andres

Faculty of Psychology and Educational Sciences, Department of Experimental Psychology, Ghent University, Henri Dunantlaan 2, 9000 Ghent, Belgium

e-mail: jeanphilippe.vandijck@ugent.be

J.-P. van Dijck

Collaborative Antwerp Psychiatric Research Institute, University of

Antwerp, Antwerp, Belgium

M. Andres

Fonds de la Recherche Scientifique, FNRS, Brussels, Belgium
Keywords Numbers $\cdot$ Space $\cdot$ Action · Grasping · Working memory $\cdot$ Embodied cognition $\cdot$ Order

\section{Introduction}

Although spatial and motor experiences are often associated with number processing, the exact nature of their interactions remains unclear. The dominant idea is that number and space interactions reflect the representation of number magnitude on a left-right oriented continuum (Dehaene, 1992; Hubbard, Piazza, Pinel, \& Dehaene, 2005). The finding that left-sided responses show a reaction time (RT) advantage over rightsided responses while judging small numbers and the reverse while judging large numbers is often taken as positive evidence for this view (i.e., SNARC; Dehaene, Bossini, \& Giraux, 1993). Further theoretical attempts have suggested that number and space rely on a generalized magnitude system that also serves sensory-motor processes, such as the transformation of object size into an appropriate hand posture for grasping (Fischer, 2012; Walsh, 2003). The rationale is that, in everyday situations, space is rarely processed separately from other magnitudes relevant for action (such as time or object size) and that consequently the learning of these coupled metrics sets the ground for a generalized magnitude system that would also be beneficial for number representation (e.g., Bueti \& Walsh, 2009). Besides number and space interactions, several studies showed that non-spatial kinematic parameters are systematically associated with number. For instance, grip aperture increases when large compared to small numbers are presented prior to the grasping movement (Andres, Davare, Pesenti, Olivier, \& Seron, 2004; Andres, Seron, \& Olivier, 2007; Chiou, Wu, Tzeng, Hung, \& Chang, 2012; Lindemann, Abolafia, Girardi, \& Bekkering, 2007). Other studies showed that small numbers are judged faster than large numbers when the response requires closing the 
index and thumb (precision grip) as for grasping a small object, whereas large numbers are judged faster than small numbers when the response requires opposing the fingers and the palm (whole-hand grip) as for grasping a large object (Chiou, Chang, Tzeng, \& Wu, 2009; Moretto \& di Pellegrino, 2008).

These stimulus-response compatibility (SRC) effects seem to support theories postulating a shared magnitude system (e.g., Bueti \& Walsh, 2009). However, another class of theories suggests that these effects do not imply a shared underlying representation but actually result from an overlap in taskrelated processes underlying decision making (e.g., Van Opstal \& Verguts, 2013). One particular instance of taskrelated process that seems to underlie number-space interactions is related to the maintenance of numbers in working memory (WM). Several data have shown that a SNARC-like effect is observed whenever a series of items - with or without magnitude information - is processed in serial WM, i.e. initial items are preferentially associated with the left and items towards the end of the sequence with right (De Belder, Abrahamse, Kerckhof, Fias, \& van Dijck, 2014; van Dijck \& Fias, 2011; van Dijck, Abrahamse, Majerus, \& Fias, 2013). This finding suggests that serial position in WM - rather than just magnitude - is responsible for number and space interactions and thereby provides a parsimonious framework to account for the context dependency of the interactions between numbers and space (van Dijck \& Fias, 2011; for a review see Fias, van Dijck \& Gevers, 2011). A recent study further showed that the instruction given to participants critically determines the interaction between the serial position of numbers in WM and response side, strengthening the idea that the interactions between number and space are determined by decision and context-related properties of the task (Ginsburg et al., 2014).

Other findings suggest that number and grip aperture interference is also sensitive to task-related aspects. For example, it has been shown that number and grip interference could be modulated on a trial-to-trial basis by manipulating the relative value of numbers (small vs. large) within the task set (e.g., 5/8 vs. 5/2; Chiou et al., 2012). Priming effects were further observed between numbers and ungraspable objects, i.e., animals, whose size congruency (small vs. large) was determined by the task set (e.g., dog/elephant vs. dog/snail; Gabay, Leibovich, Henik \& Gronau, 2013). These findings indicate the mapping of number and grasp representations is more flexible than suggested by magnitude theories and they raise the question of whether this mapping is mediated by the same serial order WM mechanisms as those identified to account for the flexibility of the relation between numbers and space.

The goal of this study is to investigate the roles of magnitude and serial order WM processes in linking numbers to grasp (precision grip vs. whole-hand grip) and space (left vs. right) representations. For this purpose, we used a WM paradigm that allowed us to orthogonally vary number magnitude and serial position within the same experiment (van Dijck \&
Fias, 2011). Participants were required to make parity judgments on a series of numbers that they had to maintain in serial order in WM during the task, while the response set was manipulated to measure interference of number magnitude and/or serial position with grasping (Experiment 1) and (unimanual) left-right movements (Experiment 2). If number and grasp interactions reflect the mapping of magnitude estimates on motor responses, the precision grip used for grasping small objects will be initiated faster in response to small numbers than to large ones, whereas the power grip used for grasping large objects will be initiated faster in response to large numbers than to small ones. On the other hand, if number and grasp interactions reflect the mapping of serial order information on motor responses, the first items in working memory will facilitate one response whereas the last items will facilitate the other response irrespective of their magnitude. Based on several previous findings, we predict that number and space interactions will reveal an association of left responses with the initial items in working memory and of right responses with the last items in working memory, even when executed uni-manually.

The results of this experimental setup will provide information for the debate between magnitude and decisional theories as different predictions can be made from both. Magnitude theories predict an interaction with number magnitude irrespective of the response set because number, space, and action are linked by a common metrics for magnitude processing. Decisional theories on the other hand assume that number, space, and grasping are linked by common computational constraints associated with the task-related processes and therefore predict more flexibility in the nature of these interactions.

\section{Experiment 1}

\section{Methods}

\section{Participants}

Twenty-four participants with normal or corrected-to-normal vision gave their informed consent to participate (mean age \pm SD: $21 \pm 6$ years; 18 females; 21 right-handed). They were all naive regarding the goals of the experiment. Three (female) participants were omitted from the analysis due to empty cells in their dataset after trimming errors.

\section{Stimuli and procedure}

The entire experiment consisted of 20 blocks. Each block was divided in three subsequent phases: an encoding phase, a phase where the parity judgment task was administered, and a phase to ensure that the entire WM sequence was memorized 
till the end of the block. The encoding phase started with the self-paced (by means of space-bar presses with the nondominant hand) sequential display of five digits that participants had to memorize in the order of presentation. The parity judgment task started 2,500 ms after the last digit offset. All digits were randomly presented twice, with the restriction that the same digit could not appear on consecutive trials. Participants were asked to respond only to the numbers that were part of the memorized sequence in order to ensure WM access. Subjects were asked to squeeze the small cylinder between the index finger and thumb (precision grip) in response to even (or odd) digits and to clamp down the other fingers over the large cylinder (whole-hand grip) in response to odd (or even) digits; the mapping between grip and parity was counterbalanced across subjects (see Fig. 1B). The experimenter initiated each trial; a digit appeared $500 \mathrm{~ms}$ after the fixation point and remained visible for $1,750 \mathrm{~ms}$ or until the participant's response that was encoded on-line by the experimenter. After the parity judgment task, participants had to identify the memorized sequence among four different sequences that were simultaneously displayed below each other by using the keyboard. The incorrect sequences differed from the correct one by a random change in the order of the elements of the memorized sequence. Twenty different WM sequences were presented after one practice sequence. Blocks in which the memorized sequence was not correctly identified were repeated at the end of the experiment. Care was taken that, over the

A

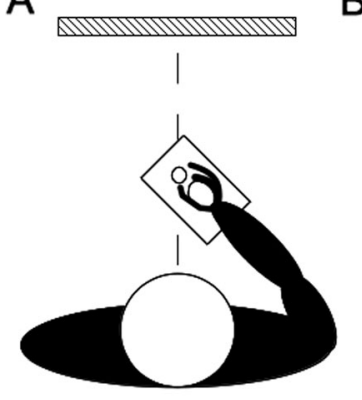

B
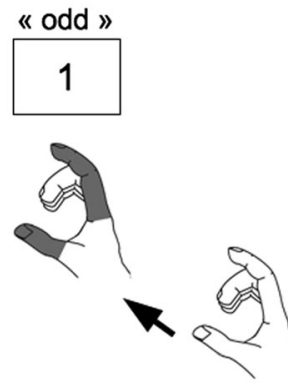

C

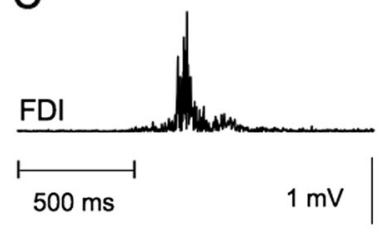

FDS

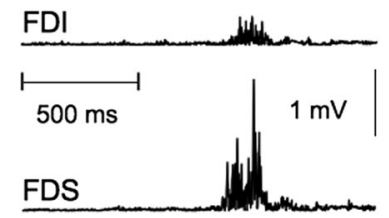

Fig. 1 (A) Upper view of the participant's posture. (B) Illustration of both response movements using one possible response assignment: odd numbers are responded to by grasping the manipulandum between the index and the thumb (i.e., precision grip), whereas even numbers are responded to by grasping the manipulandum between the other fingers and the palm (i.e., whole-hand grip). (C) Individual EMG recordings corresponding to the trials described in (B): on the left panel, the closure of the precision grip activated the FDI; on the right panel, the closure of the whole-hand grip activated the FDS entire experiment, each number appeared an equal amount of times in each position of the memorized sequence and as a nogo trial. E-prime 1.3 (Psychological Software Tools, Pittsburgh, PA, USA) was used to control the stimulus display and trigger the electromyographic (EMG) recordings.

Arabic digits (ranging from 1 to $10 ; 0.6 \times 0.5 \mathrm{~cm}$ ) were displayed in white on a black background at the center of a 17in monitor. The viewing distance was $50 \mathrm{~cm}$ and the body midline of participants was aligned with the center of the screen. Their dominant hand rested on the table on its ulnar edge, with the wrist positioned midway between pronation and supination, next to a manipulandum composed of a wooden cylinder $(3 \times 15 \mathrm{~cm})$ located $4-9 \mathrm{~cm}$ (adjusted to hand size) from a smaller wooden cylinder $(1.7 \times 15 \mathrm{~cm})$. The elbow was flexed at about $120^{\circ}$ and the forearm laid on a padded support in line with the two cylinders, forming an angle of about $60^{\circ}$ with the body. The handgrip was kept half opened between trials, with the index and thumb positioned around the small cylinder and the other fingers positioned in palm opposition around the large cylinder. Participants were instructed to leave approximately $1 \mathrm{~cm}$ between the tip of their fingers and the cylinders (see Fig. 1A).

\section{Electromyography}

EMG activity was recorded with the ActiveTwo system (BioSemi, Amsterdam, The Netherlands). Sintered $11 \times$ $17 \mathrm{~mm}$ active $\mathrm{Ag}-\mathrm{AgCl}$ electrodes were placed over the right first dorsal interosseus (FDI) and flexor digitorum superficialis (FDS) muscles in a belly-tendon arrangement. The FDI contributes to the precision grip by allowing index abduction and flexion, whereas the FDS contributes to wholehand grip by allowing flexion of the middle phalanges of all fingers at the proximal interphalangeal joints (see Fig. 1C). The EMG signal was amplified (internal gain scaling), digitized at $2 \mathrm{kHz}$, high-pass filtered at $3 \mathrm{~Hz}$, and stored on a PC for off-line analysis.

Data analysis

The dependent variable was the reaction time (RT), defined as the time interval between the stimulus presentation and the EMG burst of the agonist muscles. The EMG recordings were then rectified and the onset of the phasic EMG activity was defined as the time where EMG activity exceeded $10 \%$ of maximal activity for a continuous period of $10 \mathrm{~ms}$.

Only correct go-trials from WM blocks with accurate sequence recognition were included in the analysis. For each participant, mean RTs were computed for each condition and entered in a $5 \times 5 \times 2$ repeated measures ANOVA with numerical MAGNITUDE (levels $1-5$ corresponding to the magnitude of the numbers collapsed over five magnitudes: $[1,2]$, $[3,4],[5,6],[7,8],[9 ; 10])$, sequence ORDINAL POSITION (levels 
1-5 corresponding to the five positions in the memorized sequence) and RESPONSE (precision grip vs. whole-hand grip) as within-subject variables.

With regard to the hypotheses at hand, the two-way interaction between ORDINAL POSITION and RESPONSE, the two-way interaction between MAGNITUDE and RESPONSE, and the threeway interaction were of most interest. In order to assess linear relationships in these interactions, we computed the polynomial contrast of the difference scores (for a similar procedure, see van Dijck et al., 2013).

Results

It took on average $20.50 \pm 0.91$ blocks before all 20 sequences were correctly recognized. During parity judgment, the gono-go instructions were successfully performed in $97 \%$ (SD = $2 \%$ ) of the trials, and of the correct go-trials the parity status was judged correctly $95 \% \pm 3 \%$. Average reaction time was $954 \pm 194 \mathrm{~ms}$.

The repeated measures ANOVA revealed a main effect of ORDINAL POSITION $\left[F(4,80)=4.31, p=.003, \eta^{2}=.177\right]$. Average RTs per position were 927, 956, 943, 970, and $983 \mathrm{~ms}$. A polynomial contrast confirmed a linear trend $\left[F(1,20)=12.68, p=.002, \eta^{2}=.388\right]$, suggesting that serial order was processed (Sternberg, 1967). A clear interaction between MAGNITUDE and RESPONSE was observed $[F(4,80)=$ $\left.10.68, p<.001, \eta^{2}=.348\right]$. A polynomial contrast of MAGNITUDE in its interaction with RESPONSE revealed the presence of a linear relationship $\left[F(1,20)=37.73, p<.001 \eta^{2}=\right.$ $.654]$, indicating that precision grip responses were initiated faster in response to small than to large digits, whereas wholehand grip responses were initiated faster in response to large than to small digits. There was no interaction between ORIDINAL POSITION and RESPONSE $\left[F(4,80)=.43, p=.79, \eta^{2}\right.$ $=.021]$ and no three-way interaction $[F(16,320 ; 7.488$, $\left.149.760)^{1}=.65, p=.72, \eta^{2}=.032\right]$ (see Fig. 2).

\section{Experiment 2}

Methods

\section{Participants}

Twenty participants gave their informed consent to participate in the experiment (mean age \pm SD: $22 \pm 3$ years; 15 females; 18 right-handed). They were recruited according to the same criteria as in Experiment 1.

\footnotetext{
${ }^{1}$ Greenhouse-Geisser correction was applied when necessary and reported after the actual degrees of freedom.
}

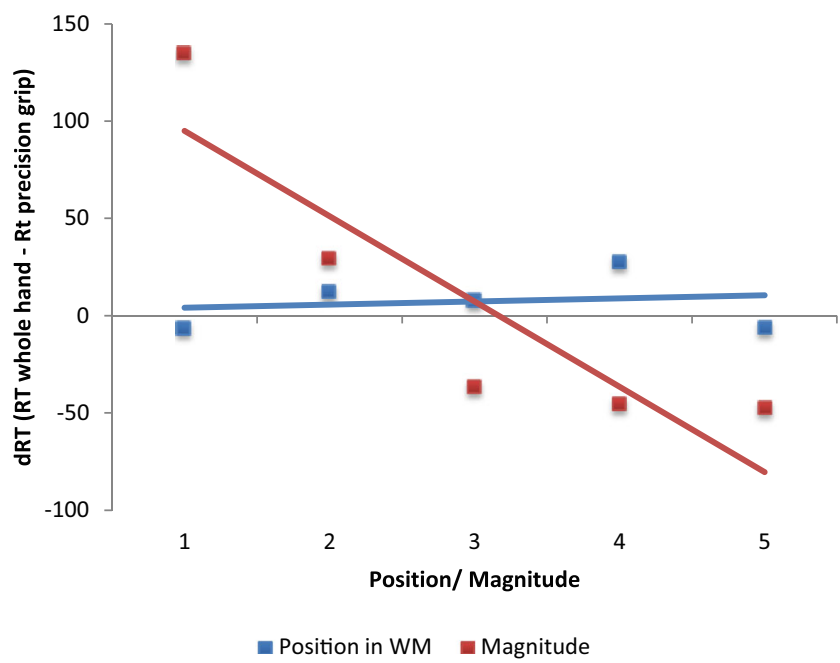

Fig. 2 Experiment 1. Reaction time (RT) differences between the two response grips as a function of numerical magnitude or serial position of the number in working memory (WM). Positive values reflect faster initiation of precision grip than whole-hand grip responses

\section{Stimuli and procedure}

The experimental setup and design were virtually identical to Experiment 1, except that participants were asked to judge the parity of the number by pressing a left or a right button with the thumb or the little finger of the dominant hand. The thumb and little finger were chosen to match the fingers involved in the two responses in Experiment 1, i.e., the precision grip (formed by the index and the thumb) and the whole-hand grip (formed by the middle finger, the ring finger, the little finger, and the palm). This response set also allowed us to control for a possible role of finger position in the SRC-effects observed in Experiment 1, knowing that Western individuals used to count from thumb to little finger as numbers increase (Lindemann, Alipour, \& Fischer, 2011). The two buttons were arranged horizontally over a response box (distance $9.5 \mathrm{~cm}$ ) aligned with the body midline of the participants. The mapping between left-right responses and odd-even numbers was counterbalanced across participants; the response deadline was set to $1,500 \mathrm{~ms}$ and the ITI to $1,000 \mathrm{~ms}$.

\section{Results}

It took on average $20.25 \pm 0.55$ blocks before all 20 sequences were correctly recognized. During parity judgment, the gono-go instructions were successfully performed in $97 \% \pm 3 \%$ of the trials, and of the correct go-trials the parity status was judged correctly $95 \% \pm 3 \%$. Average reaction time was $789 \mathrm{~ms} \pm 13 \mathrm{~ms}$.

The repeated measures ANOVA revealed a main effect of MAGNITUDE $\left[F(4,76)=3.29, p<.015, \eta^{2}=.148\right]$ and of ORDINAL POSITION $[F(4,76 ; 2.871,54.556)=15.38, p<.001$, $\left.\eta^{2}=.447\right]$. Average RTs per magnitude were 819, 792, 801, 
789, and 769 ms. Average RTs per position were 759, 765, 798,820 , and $828 \mathrm{~ms}$. A polynomial contrast confirmed a linear trend in the effect of MAGNITUDE and POSITION [ both $F(1$, 19) $\left.>13.11, p{ }^{\prime}<.002, \eta^{2}{ }^{\prime} s>.40\right]$, suggesting processing of numerical magnitude and serial order in WM. A clear interaction between ORDINAL POSITION and RESPONSE was observed $\left[F(4,76)=8.63, p<.001, \eta^{2}=.312\right]$ and a polynomial contrast of ORDINAL POSITION in its interaction with RESPONSE revealed the presence of a linear relationship $[F(1,29)=30.95, p<.001$ $\eta^{2}=.620 ;$, indicating faster left button presses in response to early than to late WM elements and faster right button presses in response to late than to early WM elements. There was no evidence for an interaction between MAGNITUDE and RESPONSE $\left[F(4,76 ; 2.549,48.440)=.47, p=.67, \eta^{2}=.024\right]$ and no threeway interaction $\left[F(16,304)=1.48, p=.11, \eta^{2}=.072\right]$ (see Fig. 3).

\section{Omnibus analysis}

To investigate whether the reversed data-pattern of Experiment 1 and Experiment 2 reflect a true double dissociation, a final omnibus analysis was carried out. For this purpose, the regression weights of the MAGNITUDE $\times$ RESPONSE and ORDINAL POSITION $\times$ RESPONSE interactions of both experiments were entered into a repeated measures ANOVA with EFFECTS as within-subject and EXPERIMENT (Experiment 1 and Experiment 2) as between-subject factors. This ANOVA statistically supported the presence of a double dissociation as the interaction between EFFECTS and EXPERIMENT was significant $\left[F(1,39)=32.48, p<.001, \eta^{2}=.45\right]$.

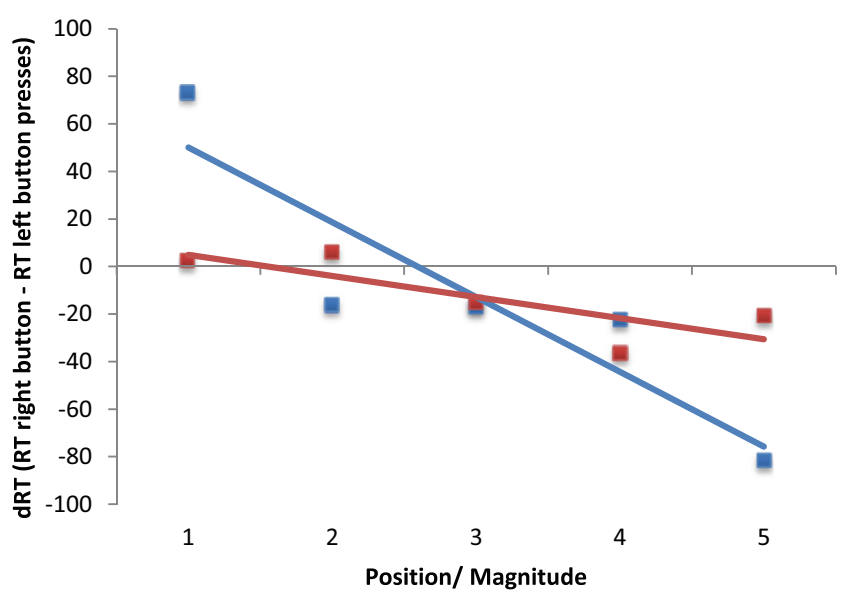

Eosition in WM Magnitude

Fig. 3 Experiment 2. Reaction time (RT) differences between right and left responses as a function of numerical magnitude or position of the number in working memory (WM). Positive values reflect faster initiation of left than right responses

\section{Discussion}

The present study aimed to investigate the role of magnitude and serial order processing in linking numbers to grasp (precision grip vs. whole-hand grip) and space (left vs. right) representations. Our paradigm required participants to make parity judgments on numbers between 1 and 10 while maintaining them in a distinct order in WM for the sake of a dual task. Experiment 1 showed that precision grips were initiated faster than whole-hand grips after the presentation of a small number and the reverse after the presentation of a large number, irrespective of the position of numbers in WM. Experiment 2 showed that left responses were initiated faster than right responses after the presentation of a number kept in first position in WM and the reverse after the presentation of a number kept in final position in WM, irrespective of number magnitude. The finding that SRC-effects were selective to one dimension in each experiment does not result from a failure to process the other dimension. Experiment 1 evidenced a linear increase of RTs with serial position, which is considered to be a hallmark observation of serial order processing (e.g., Marshuetz, 2005). This main effect of position, however, did not interact with grasping movements. Experiment 2 revealed a linear effect of number magnitude, which suggests that this dimension was processed as well but no interaction with leftright movements was observed. Note that this effect of magnitude reflects higher RTs to small numbers, which is reversed compared to the size effect (i.e., the observation that RTs for small numbers are generally speaking lower compared to large numbers, e.g, Buckley \& Gillman, 1974) sometimes observed in other studies. Importantly, however, such a reversed size effect has already been observed in WM paradigms highly similar to the one used here (e.g., van Dijck \& Fias, 2011; van Dijck et al., 2014) and thus suggests being a robust observation. Future work is needed to understand why the effect of magnitude reverses when the numbers are in WM.

These findings lead us to the conclusion that distinct dimensions underlie the overlap of numbers with grasping and with spatially defined responses. Dimensional overlap occurs when a mapping exists between a stimulus set and a response set that preserves the relations and operations defining the structure of the stimuli with respect to one dimension (Kornblum, Hasbroucq, \& Osman, 1990). Experiment 1 suggests that interference of numbers with grip selection originates from a common relational system that discriminates numbers or grips with respect to magnitude. The categorization of handgrips along a magnitude dimension relates to the preferential use of a precision grip for grasping small objects and a whole-hand grip for grasping large or heavy objects. Experiment 2 suggests that the interference of numbers with left-right movements is observed because these responses entail spatial relations that also underlie the coding of numbers with respect to their serial position in WM. More generally, 
these results validate the use of SRC effects to assess dimensional overlap between cognitive and sensory-motor processes (Fias, Lauwereyns, \& Lammertyn, 2001).

Previous observations have shown that our ability to grasp an object is overestimated (or underestimated) when the object is preceded by the presentation of small (or large) numbers, whereas such a bias is not observed when the object is preceded by the presentation of the first (or last) letters of the alphabet (Badets, Andres, Di Luca, \& Pesenti, 2007). However, the lack of explicit instructions to process alphabetical order in this study may have decreased the saliency of order information during grasping judgments (Gevers, Reynvoet, \& Fias, 2003). Our WM task ascertains that serial order is explicitly processed, thereby providing a better paradigm to measure the interference of number magnitude and serial order with different response sets. Hence, it was previously used to reveal an association between the serial position of numbers in WM and the spatial location of response keys (van Dijck \& Fias, 2011) or visual targets in a detection task (van Dijck et al., 2013; 2014). The present study replicates this association with unimanual responses and further shows that the determinant of number-space interactions (i.e., the spatialposition association) is not the WM paradigm (where serial order processing is made obligatory) since an interaction between number magnitude and grasping responses was observed while keeping the requirement to process serial position in WM.

A long-standing issue concerns the inter-dependence of magnitude (e.g., which number is the largest?) and serial order processing (e.g., which number comes first?) in numerical tasks. Previous studies revealed different behavioral (Turconi, Campbell, \& Seron, 2006) and neural signatures (Turconi, Jemel, Rossion, \& Seron, 2004; Zorzi, Di Bono, \& Fias, 2011) while participants judged these two dimensions. The finding that number magnitude and serial order induce distinct SRC effects in parity judgments provides further evidence that these two dimensions are processed separately. It is worth noting that, in our study, serial order processing was defined as a component of WM but numbers also benefit of a learned representation of order in long-term memory (LTM), like alphabetical or calendar order (Gevers et al., 2003). The question of how the LTM representation of numerical order interacts with the contents of WM remains to be investigated.

The present study thus provides direct empirical evidence for a dissociated overlap of grasping and left-right movements with number magnitude and serial order processing and put important constraints to theories explaining these interactions. Theories assuming a common metric for magnitude processing underlying interactions between number, space, and action (Fischer, 2012; Walsh, 2003) and decisional theories (e.g., Van Opstal \& Verguts, 2013) have difficulties in explaining how different actions can lead to qualitative different interactions. From the first class of theories, interactions with only the magnitude dimension would have been predicted, as serial order is typically not considered to be a special instance of a magnitude representation. Here we clearly show that numbers are represented across multiple dimensions and that each dimension interferes with the responses as a function of the task set. We argue therefore that to come to a fuller understanding, future updates of these theories should consider serial order (whether or not in WM) as an important dimension of numbers. Decisional theories on the other hand should further specify how a simple change of the response set, leaving all task- and decision-related aspects unchanged, results in qualitative different interactions.

In sum, the current study provides the first direct evidence that grasp and space representations are related to different aspects of number processing, implying a functional distinction between magnitude and serial order processing. More generally, the current results provide a clear illustration that stimulus-response interactions are determined by the dimensional overlap of stimulus and response properties and point to the importance to integrate these ideas in theoretic and computation models on the relation between numbers, space, and action to come to a fuller understanding of the cognitive dynamics underlying them.

Acknowledgments This work was supported by the Ghent University Multidisciplinary Research Partnership "The integrative neuroscience of behavioral control" and by grant P7/11 from the Interuniversity Attraction Poles program of the Belgian federal government. MA is a Research Associate at the FNRS-FRS (Belgium).

\section{References}

Andres, M., Davare, M., Pesenti, M., Olivier, E., \& Seron, X. (2004). Number magnitude and grip aperture interaction. Neuroreport, 15(18), 2773-2777.

Andres, M., Seron, X., \& Olivier, E. (2007). Contribution of hand motor circuits to counting. Journal of Cognitive Neuroscience, 19(4), 563 576.

Badets, A., Andres, M., Di Luca, S., \& Pesenti, M. (2007). Number magnitude potentiates action judgements. Experimental Brain Research, 180, 525-534.

Buckley, P. B., \& Gillman, C. B. (1974). Comparison of digits and dot patterns. Journal of Experimental Psychology, 103, 1131-1136.

Bueti, D., \& Walsh, V. (2009). The parietal cortex and the representation of time, space, number and other magnitudes. Philosophical Transactions of the Royal Society of London Series B-Biological Sciences, 364, 1831-1840.

Chiou, R. Y. C., Chang, E. C., Tzeng, O. J. L., \& Wu, D. H. (2009). The common magnitude code underlying numerical and size processing for action but not for perception. Experimental Brain Research, 194, $553-562$

Chiou, R. Y. C., Wu, D. H., Tzeng, O. J. L., Hung, D. L., \& Chang, E. C. (2012). Relative size of numerical magnitude induces a size-contrast effect on the grip scaling of reach-to-grasp movements. Cortex, 48(8), 1043-1051.

De Belder, M., Abrahamse, E.L., Kerckhof, E., Fias, W. \& van Dijck, J.-P. (2014). Serial position markers in space: Visuospatial priming of serial order working memory retrieval. PLoS One. in press. 
Dehaene, S. (1992). Varieties of numerical abilities. Cognition, 44(1-2), $1-42$.

Dehaene, S., Bossini, S., \& Giraux, P. (1993). The mental representation of parity and number magnitude. Journal of Experimental Psychology-General, 122(3), 371-396.

Fias, W., Lauwereyns, J., \& Lammertyn, J. (2001). Irrelevant digits affect feature-based attention depending on the overlap of neural circuits. Cognitive Brain Research, 12(3), 415-423.

Fias, W., van Dijck, J. P., \& Gevers, W. (2011). How number is associated with space? The role of working memory. In E. Brannon \& S. Dehaene (Eds.), Space, time and number in the brain - searching for evolutionary foundations of mathematical thought: Attention and performance XXIV (pp. 133-148). Amsterdam: Elsevier Science.

Fischer, M. H. (2012). A hierarchical view of grounded, embodied, and situated numerical cognition. Cognitive Processing, 13(Suppl 1), S161-S164.

Gabay, S., Leibovich, T., Henik, A., \& Gronau, N. (2013). Size before numbers: Conceptual size primes numerical value. Cognition, 129, $18-23$.

Gevers, W., Reynvoet, B., \& Fias, W. (2003). The mental representation of ordinal sequences is spatially organized. Cognition, 87(3), B87-B95.

Ginsburg, V., van Dijck, J.-P., Previtali, P., Fias, W., \& Gevers, W. (2014). The impact of verbal working memory on number-space associations. Journal of Experimental Psychology. Learning, Memory, and Cognition, 40(4), 976-986.

Hubbard, E. M., Piazza, M., Pinel, P., \& Dehaene, S. (2005). Interactions between number and space in parietal cortex. Nature Reviews Neuroscience, 6(6), 435-448.

Kornblum, S., Hasbroucq, T., \& Osman, A. (1990). Dimensional overlap: Cognitive basis for stimulus-response compatibility. Psychological Review, 97(2), 253-270.

Lindemann, O., Abolafia, J. M., Girardi, G., \& Bekkering, H. (2007). Getting a grip on numbers: Numerical magnitude priming in object grasping. Journal of Experimental Psychology: Human Perception and Performance, 33(6), 1400-1409.
Lindemann, O., Alipour, A., \& Fischer, M. H. (2011). Finger counting habits in Middle Eastern and Western individuals: An online survey. Journal of Cross-Cultural Psychology, 42(4), 566-578.

Marshuetz, C. (2005). Order information in working memory: An integrative review of evidence from brain and behavior. Psychological Bulletin, 131(3), 323-339.

Moretto, G., \& di Pellegrino, G. (2008). Grasping numbers. Experimental Brain Research, 188, 505-515.

Sternberg, S. (1967). Retrieval of contextual information from memory. Psychonomic Science, 8(2), 55-56.

Turconi, E., Campbell, J. I. D., \& Seron, X. (2006). Numerical order and quantity processing in number comparison. Cognition, 98, 273-285.

Turconi, E., Jemel, B., Rossion, B., \& Seron, X. (2004). Electrophysiological evidence for differential processing of numerical quantity and order in humans. Cognitive Brain Research, 21, 22-38.

van Dijck, J.-P., \& Fias, W. (2011). A working memory account for spatial numerical associations. Cognition, 119(1), 114-119.

Van Opstal, F., \& Verguts, T. (2013). Is there a generalized magnitude system in the brain? Behavioral, neuroimaging, and computational evidence. Frontiers in Psychology, 4, article 435.

van Dijck, J.-P., Abrahamse, E. L., Majerus, S., \& Fias, W. (2013). Spatial attention drives serial order retrieval in verbal working memory. Psychological Scienc, 24(9), 1854-1859.

van Dijck, J.-P., Abrahamse, E. L., Acar, F., Ketels, B., \& Fias, W. (2014). A working memory account of the interaction between numbers and spatial attention. Quarterly Journal of Experimental Psychology, 67(8), 1500-1513.

Walsh, V. (2003). A theory of magnitude: Common cortical metrics of time, space and quantity. Trends in Cognitive Sciences, 7(11), 483488.

Zorzi, M., Di Bono, M. G., \& Fias, W. (2011). Distinct representations of numerical and non-numerical order in the human intraparietal sulcus revealed by multivariate pattern recognition. NeuroImage, 56, 674680. 